\title{
Reminiscence and Arousal: A Connectionist Model
}

\author{
Eric Chown (echown@bowdoin.edu) \\ Department of Computer Science, 8650 College Station \\ Brunswick, ME 04011 USA
}

\begin{abstract}
In recall tasks, increased levels of arousal soon after presentation time leads to short-term performance that is contradictory to standard memory models. Despite the fact that long-term recall is excellent in such situations, short-term recall is poor, worse than in the long-term case. This article presents a model, based upon Hebb's cell assembly construct, to account for this puzzling data. The system, called MultiTrace, has previously been used to model a lexical priming task and was adapted with only minor changes for this task.
\end{abstract}

\section{Introduction}

The relationship between arousal and short-term memory has presented a problem for standard memory models for nearly 40 years. In a pair of studies in the early 1960s Kleinsmith and Kaplan $(1963 ; 1964)$ found that while there is a positive correlation between increases in arousal and long-term memory, in the short-term case the reverse is true. Further, in cases of where arousal markedly increases, short-term recall is substantially worse than long-term recall, an effect called "reminiscence." Because these results challenge standard memory models in which short-term memory is necessarily stronger than long-term memory, they have often been criticized, but just as often have been replicated (Eysenck, 1977; Weingartner \& Parker, 1984; Revelle \& Loftus, 1990). To date, no widely accepted explanation has ever been provided to account for the data (see, for example, Revelle \& Loftus, 1990). However, more modern views of memory, updated with an increased understanding of the underlying neural mechanisms involved, can now provide a plausible explanation of the data. This article presents an existing model that can account for the data in a way that is surprisingly close to the original account of Kleinsmith and Kaplan.

The model used in this article, called MultiTrace (Sonntag, 1991; Chown, 1994; Forbell \& Chown, 2000), is a variant on the cell assembly construct proposed by Hebb (1949) and later extended by Kaplan, et. al. in their TRACE system (1991). What makes the cell assembly model so attractive for this task is that, unlike many other neural network models, cell assemblies have complex temporal dynamics directly affected by the physiological properties of neurons. MultiTrace is an extension of the single cell assembly
TRACE model to allowing sequence learning through the inclusion of multiple cell assemblies.

This article will begin with a discussion of the Kleinsmith and Kaplan data and some of the theoretical problems it raises. Next the MultiTrace model is presented, highlighting parts of the model directly relevant to this task. Finally, the original experiments are modeled in MultiTrace and the results are compared to the originals.

\section{Reminiscence}

The learning paradigm used by Kleinsmith and Kaplan was a simple paired-associate task. In the first experiment, subjects were shown a word for four seconds and then the same word and a number for another four seconds. The recall task was to remember the number when the word was given as a cue. Changes in galvanic skin response (GSR) deflection were used as the measure of arousal response. These changes were sorted into "high" and "low" categories for each subject. In the long-term case (one week), Kleinsmith and Kaplan got the results that they expected - recall was better for words in the "high" category. The surprising result was that in the shortterm cases ( 2 minutes and 20 minutes) this was not the case, and further, recall in the "high" categories was worse than in the long-term case. When the arousal deflection was "low", the recall curves generated were what would normally be predicted - short-term recall was very good but decayed over time (Figure 1). In the "high" case, however, Kleinsmith and Kaplan found that the recall curve was essentially an inverted $U$ (Figure 1). To address criticism of the original study, Kleinsmith and Kaplan replicated their work the following year, but used nonsense syllables instead of words, and got qualitatively similar results.

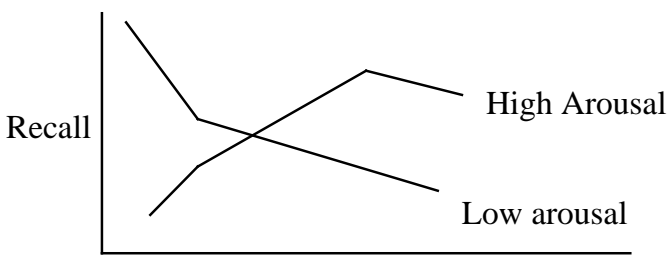

Log Time

Figure 1: The Kleinsmith and Kaplan data. 
The major question raised by the Kleinsmith and Kaplan data is why recall is so poor in the short-term case in the high deflection condition. Among other things, the results would seem to discount the notion that long-term memory is just a decayed version of short-term memory, or that memories transition from short-term into long-term storage.

Kleinsmith and Kaplan proposed that the neural circuits involved in the task rapidly reverberated under conditions of increased arousal and were relatively unavailable because of that reverberation. They went on to theorize that this same reverberation was responsible for the better long-term recall due to greater perseverative consolidation. This explanation was discounted due largely to the lack of an accepted mechanism that could account for the unavailability of the circuits in the short-term. Kleinsmith and Kaplan proposed neural fatigue as the mechanism, but this is a property of neurons that only started to become accepted more than 20 years later (Ito, 1992; Artola \& Singer, 1993) and was criticized at the time for being implausible.

Most of the alternative explanations proposed in the interim involved some combination of consolidation and interference in conjunction with separate short and long-term memory mechanisms. None of them were satisfactory (Revelle \& Loftus, 1990), and work in the area appears to have died out due to the lack of attractive theories. The model presented here is closely related to Kleinsmith and Kaplan's original proposal. The neural circuits in this case are cell assemblies. In this paradigm learning comes as the result of correlated neural activity (this is usually called Hebbian learning). One effect of an increase in arousal is to generate intense and focused activity in the brain (Oades, 1985). In a Hebbian paradigm one byproduct of intense activity is that the areas of focused activity experience more correlated activity and therefore more learning. As this activity stretches out over a few seconds it essentially serves as the memory consolidation period. On the other hand, this intense activity also tends to fatigue the neurons that have been repeatedly firing. The net result is that these fatigued cell assemblies temporarily require an unusual amount of stimulation in order to become reactivated.

\section{The MultiTrace Model}

Hebb developed the cell assembly construct to address questions concerning the temporal nature of neural processing. Hebb needed a way to explain how neurons could hold information over time (e.g. the psychological concept of "set") even though they essentially pass through information. He solved this problem by proposing that cell assemblies consist of a large collection of neurons that are highly interconnected. These connections form a kind of loop that enables the cell assembly to effectively hold information through the reverberation of the loop once it becomes active. In this way cell assemblies are neural analogs of "symbols." Hebb's theory was rejected after early models showed that the recurrent connections tended to lead to out of control activity (Rochester, et. al., 1956). This was because Hebb had cautiously omitted inhibition from his model because no direct physiological evidence existed for it at the time. More recently, however, cell assembly theory has undergone something of a revival as experimental evidence for their existence has been found (Amit, 1995) and several models have been proposed that extend Hebb's original conception (Kaplan, et al, 1991; Hetherington \& Shapiro, 1993; Amit, 1995; Horn, et al., 2000).

\section{TRACE}

MultiTrace is based upon one such model, the TRACE (Tracing Recurrent Activity in Cognitive Elements) model of Kaplan et. al. (1991). TRACE is based on the idea that there must be counterbalancing forces to offset the tendency of cell assemblies to continually reverberate. The most important of these forces are inhibition, which provides a mechanism for selection and for competition with other cell assemblies, and fatigue which ensures that cell assemblies do not stay active indefinitely.

TRACE is modeled as a set of difference equations that capture the biological properties of the population of neurons that comprise the cell assembly. The equations are similar to population models used by biologists. Rather than trying to work out the interactions of neurons individually, the Kaplan group decided to work at a slightly higher level in order to understand their collective behavior. TRACE units, therefore, are more complex than typical neural network units, but are still at a higher level than neurons.

The crux of any cell assembly model is that activity in a cell assembly is the basis of cognitive processing. Perception, for example, would correspond to a cell assembly reaching some internal threshold of activity (defined as the percentage of active neurons at any given time step). TRACE added several theoretical constructs to Hebb's theory that are relevant to this article; they are inhibition, fatigue, and short-term connection strength.

Inhibition serves numerous roles in cognition, including selection and providing a mechanism for perceptual competition. These will be discussed in more detail in the discussion of the MultiTrace extension to TRACE.

Fatigue provides a kind of shut-off mechanism to cell assemblies, ensuring that the cognitive system does not literally get stuck on a single thought. It has also been 
speculated that fatigue may play a central role in learning in much the same way that muscle fatigue is crucial to getting stronger. Neurons, like muscles are physical systems that require fuel (e.g. transmitter substances) to work efficiently. With extreme usage that fuel supply can run out and essentially damage the system. With muscles it is the repair of the damage that makes the muscle stronger. It may be that neural fatigue is a kind of signal to rewire synapses in order for the system to run more efficiently in the future.

The important function of short-term connection strength with regard to this article, is that it provides a mechanism for short-term memory. Short-term connection strength (STCS) is based on post-tettanic potentiation, the property of neurons that once they fire they are temporarily more likely to fire again. In other words, once a cell assembly has been active, for a short time it will be easily reactivated (or recalled). STCS's counterpart in TRACE is long-term connection strength (LTCS) which comprises the long-term structure of the brain and is what changes with learning.

The TRACE equations are shown in Table 1. As difference equations, they are updated on each time step (set at 10 milliseconds in TRACE).

Table 1: The TRACE equations

\begin{tabular}{l|l} 
Update Equations & Delta Equations \\
\hline $\mathrm{A}(\mathrm{t}+1)=\mathrm{A}(\mathrm{t})+\Delta \mathrm{A}$ & $\Delta A=P-M$ \\
$\mathrm{~F}(\mathrm{t}+1)=\mathrm{F}(\mathrm{t})+\Delta \mathrm{F}$ & $P=(A+\bar{A} I) \bar{A} V$ \\
$\mathrm{~S}(\mathrm{t}+1)=\mathrm{S}(\mathrm{t})+\Delta \mathrm{S}$ & $M=\left(A^{\theta_{L}}+A \bar{A}^{\theta_{C}}\right) \bar{V}$ \\
$\mathrm{~L}(\mathrm{t}+1)=\mathrm{L}(\mathrm{t})+\Delta \mathrm{L}$ & $\Delta \mathrm{F}=\phi_{\mathrm{g}} \mathrm{A} \bar{F}-\phi_{\mathrm{d}} \mathrm{F}$ \\
& $\Delta \mathrm{S}=\sigma_{\mathrm{g}} \mathrm{A} \bar{S}-\sigma_{\mathrm{d}} \mathrm{S}$ \\
& $V=1 / v(S+L) \bar{F} \mathrm{~A}_{\mathrm{R}}$ \\
& $I=I^{\text {exc }}-I^{\text {inh }}($ see text $)$ \\
\hline$\theta_{\mathrm{l}}:$ unit loss & $\mathrm{A}:$ activity \\
$\theta_{\mathrm{c}}:$ inhibitory & $\mathrm{F}:$ neural fatigue \\
competition & $\mathrm{S}:$ STCS \\
$\mathrm{v}:$ normal factor & $\mathrm{L}:$ LTCS \\
$\phi_{\mathrm{g}}:$ fatigue growth & $\mathrm{I}:$ network input \\
$\phi_{\mathrm{d}}:$ fatigue decline & A $:$ Arousal \\
$\sigma_{\mathrm{g}}:$ STCS growth & Equation Note: $\bar{X}$ denotes \\
$\sigma_{\mathrm{d}}:$ STCS decline & \multicolumn{1}{|c}{ quantity $(1-\mathrm{X})$}
\end{tabular}

\section{MultiTrace}

MultiTrace extends TRACE by putting TRACE units into a larger architecture consisting of multiple cell assemblies. In addition to the properties of the individual units that come from the TRACE model, units also have properties associated with the architecture of the brain. There are two properties that are important with regard to this article and they have previously been used with MultiTrace to model a lexical priming task (Forbell \& Chown, 2000). The first property is what Kinsbourne (1982) called "the functional distance principle." This is the property that similar concepts tend to interfere with each other more than dissimilar ones. In neural terms, similar concepts are processed near each other in the brain and will tend to inhibit each other. This provides a means for perceptual competition among other things. The second property is that cortex consists of a large number of layers. It is possible to think of these layers vertically, with the perceptual layer on the bottom and more abstract layers higher in the hierarchy. In the original TRACE paper it was proposed that each layer consists of cell assemblies with different temporal properties (in the context of the model this is achieved by varying the parameter settings).

Figure 2 is drawn from (Forbell \& Chown, 2000) and demonstrates both of these properties. The lexical layer is more abstract than the phoneme layer and is therefore at a higher level in the cognitive hierarchy. In this case the lexical layer is one level up since words consist of sequences of phonemes. Each layer has slightly different properties (for example, perceptual layers will need to recover from fatigue very quickly so the perceptual system can always be ready for the next input) and similar items within a layer tend to compete with each other through lateral inhibition. In the figure, for example, "BLAST" will inhibit "BLACK" more than it will inhibit "BLUES" since it is more similar to "BlACK."

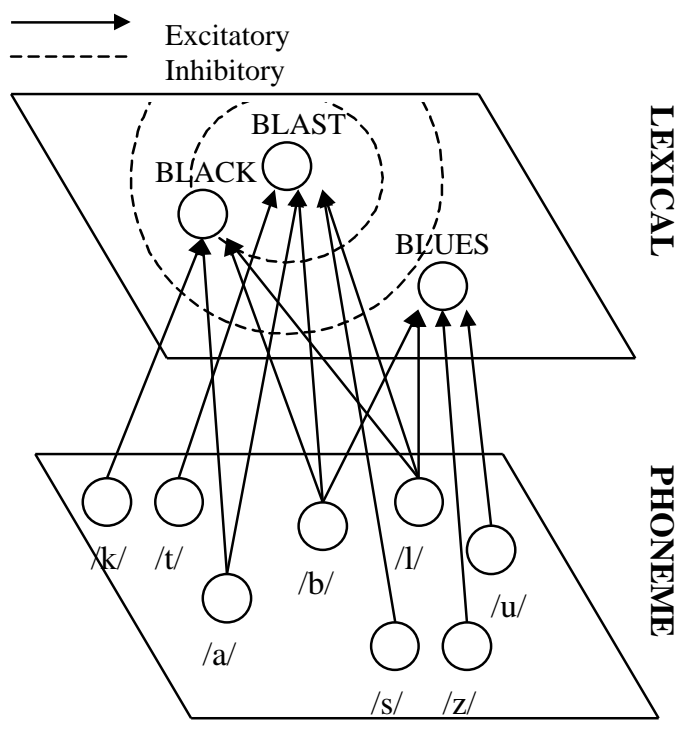

Figure 2: The two-tiered connectionist architecture. Similar words are processed near each other and will tend to inhibit each other based upon distance. 
The main extensions to the original TRACE model added in MultiTrace is to use a more complex input function that combines several sources of inhibition as well as excitation. The input equations found here are the same as in (Forbell \& Chown, 2000) and more details on the derivations can be found there.

For clarity a unit's input can be divided into inhibitory and excitatory components. Because of the functional distance principle, neighboring units will tend to inhibit each other. The amount of inhibition is a function of the distance between units and the activity and fatigue of the inhibiting unit:

$$
\begin{aligned}
I_{j k}^{i n h}= & \frac{A_{j}\left(1-F_{j}\right)}{D_{j k}} \\
& \text { j: source unit } \\
& \text { k: target unit } \\
& \text { D: distance }
\end{aligned}
$$

There is an additional inhibitory factor in the model termed "regional inhibition" which is designed to limit the total activity in any given cognitive layer. Therefore the total inhibition for a unit, $\mathrm{k}$, is the sum of the inhibition from other units, plus its regional inhibition:

$$
\begin{aligned}
I_{k}^{i n h}= & \frac{1}{L}\left(\sum_{j=1}^{n} I_{j k}^{i n h}\right)+R\left(\sum_{i=1}^{n} A_{i}\right) \\
& \text { n: number of units in a layer } \\
& \text { R: regional inhibition factor } \\
& \text { L: lateral inhibition factor }
\end{aligned}
$$

Excitatory input is computed in a conventional connectionist manner. The only difference is that units have both long-term and short-term connections between them:

$$
\begin{aligned}
& I_{j k}^{e x c}=\left(L T C S_{j k}+S T C S_{j k}\right) A_{j} \\
& I_{k}^{e x c}=\sum_{i=1}^{n} I_{j k}^{e x c}
\end{aligned}
$$

$\mathrm{j}$ : source unit, $\mathrm{k}$ : target unit $\mathrm{n}$ : number of incoming connections for unit $k$

Once the excitatory input is computed it is run through a standard sigmoid function to force it to values between 0 and 1 .

Learning in MultiTrace is based upon the Hebbian learning rule that connections between units are increased when they fire nearly simultaneously. This is modulated by the fatigue (raised to the power $P$ ) of the unit as well as a learning rate, $\Lambda$.

$$
\Delta \mathrm{LTCS}_{\mathrm{kj}}=\Lambda * \mathrm{~A}_{\mathrm{k}} * \mathrm{~A}_{\mathrm{j}} * \mathrm{~F}_{\mathrm{k}}^{\mathrm{P}}
$$

\section{Simulation and Results}

The experiments simulated in this article are from (Kleinsmith \& Kaplan, 1964). The only changes to MultiTrace from (Forbell \& Chown, 2000) were in parameter settings related to operating at a different level of cognition than the perceptual experiments in that work. The parameters internal to units are largely based upon those in the original TRACE paper (Kaplan, et. al., 1991) since it was meant to model higher cognition. With regard to the experiments, the important parameters are the ones affecting fatigue, short-term connection strength and arousal. The values are shown in Table 2. With regard to arousal, the value shown is taken to be a baseline that varies experimentally as described below.

Table 2: Unit Parameters

\begin{tabular}{|c|l|l|}
\hline Parameter & Description & Value \\
\hline$\Phi_{\mathrm{g}}$ & Fatigue Growth & 0.007 \\
\hline$\Phi_{\mathrm{d}}$ & Fatigue Decline & 0.0001 \\
\hline$\sigma_{\mathrm{g}}$ & STCS Growth & 0.1 \\
\hline$\sigma_{\mathrm{d}}$ & STCS Decline & 0.001 \\
\hline $\mathrm{A}_{\mathrm{r}}$ & Arousal & 0.95 \\
\hline
\end{tabular}

In terms of the architecture selected, a two-tiered system was used similar to the one shown in Figure 2. The reasoning is that nonsense syllables were selected specifically to be unlike normal words. This means there should be no perceptual competition between the nonsense syllables and the associated digits. Further, connections between the layers should be relatively uniform and weak. One difference between the layers is that nonsense syllables represent "unlearned" or weakly connected cell assemblies. Digits, on the other hand, are frequently used, and thus the cell assemblies that represent them should be tightly connected. In the original TRACE paper it was proposed that unlearned cell assemblies have an internal long-term connection strength (LTCS) of 0.2, while well-learned ones have an LTCS of 0.5. In the experiments that follow, this is the only difference between the two layers.

The basic format of a given run of a simulation is simple: a unit was randomly selected from the first layer and was stimulated. Meanwhile, a starting arousal level was also generated. Then two seconds later a random arousal deflection was generated. This was categorized as either "high" or "low" by comparing it against the median arousal level. Two seconds after that the original unit was again stimulated along with a randomly selected unit from the second layer. In the short-term case the network was then allowed to run for two minutes of simulated time. Then arousal was again set randomly (it was found that there was no correlation between arousal at presentation and arousal at recall 
(Kaplan \& Kaplan, 1970)) and the first unit was restimulated. If the second unit became active due to stimulation from the first, it was categorized as successful recall. The procedure was the same in the long-term case except that factors such as fatigue and STCS were simply reset to normal levels to simulate the passage of time. In addition, the network learned (in the form of changes in inter-unit LTCS) in the longterm, but not the short-term case. This is because consolidation data shows that the physical process of long-term memory generally takes at least 20 minutes Miller \& Marlin, 1984).

The 20-minute recall case was dropped for a variety of reasons. One reason is that the simulation essentially runs in real time. This means it is not possible to run a significant number of trials as compared to the 1000 trials in the other cases. A second reason is that the 20minute case mixes short-term and long-term memory processes. There is no a priori way of knowing how much the recall rates at those times are due to either factor. The tested cases are purely short-term memory on the one hand, and purely long-term memory on the other, and therefore can generate more meaningful results. There is no obvious way to set the learning rate in the 20-minute case and trying to determine one experimentally would be extremely difficult due to the time-scale involved.

The architectural parameters relating specifically to MultiTrace are given in Table 3.

Table 3: MultiTrace Parameter Settings

\begin{tabular}{|c|l|l|}
\hline Parameter & Description & Value \\
\hline $\mathrm{R}$ & Regional Inhibition & 0.1 \\
\hline $\mathrm{L}$ & Lateral Inhibition & 3 \\
\hline$\Lambda$ & Learning Rate & 0.03 \\
\hline $\mathrm{P}$ & Fatigue Power & 3 \\
\hline
\end{tabular}

To test the robustness of the model, in each simulation run a new "subject" was generated by randomly perturbing all of the relevant model parameters. For example, if the parameter's ideal value was set at 0.1 a new one was generated for each run by using a Gaussian distribution with a mean of 0.1 and a standard deviation of 0.01 . In all, 1000 runs were done for both the long and the short-term cases. The results are shown in Table 4.

Table 4: Recall Rates

\begin{tabular}{|c|c|c|}
\hline Arousal & 2-minute & 1-week \\
\hline Low & 0.55 & 0.25 \\
\hline High & 0.11 & 0.50 \\
\hline
\end{tabular}

The results are similar to those obtained by Kleinsmith and Kaplan, though exact comparisons cannot be drawn since they published curves, but not the actual numbers. Further, pursuit of an exact match is probably a fruitless enterprise as the results were later reinterpreted with different scoring methodologies (Kaplan \& Kaplan, 1970). The conclusion of that study was that the trends in the results were consistent across experiments and scoring, but with a fair degree of variation. The important trends being that initial recall is high in the low arousal case and then declines, and that the reverse is true in the high arousal case.

It is important to emphasize that the parameters for this work were drawn from previous sources. The TRACE parameters in Table 2 were taken directly, without modification, from an extension to TRACE where arousal was added (Chown, 1994). These parameters are different than those used in (Forbell \& Chown, 2000) due to the fact that different levels of cognition were being modeled. In the previous case the individual units represented phonemes; in the current case the cell assemblies represent entire syllables. Since syllables are comprised of sequences of phonemes the time course of activity must be longer. In terms of the MultiTrace architectural parameters in Table 3, the only parameter that varied from (Forbell \& Chown, 2000) was the learning rate. It is reasonable to believe that the learning rate for lexical material is different than the learning rate at higher levels of cognition, such as at the word level.

\section{Discussion}

This work serves two purposes. First, an existing biologically grounded model was used to address a theoretical problem in the memory and arousal literature. The results support Kleinsmith and Kaplan's theory that their data can be explained in terms of reverberating neural circuits. Second, their data provides further constraints in exploring the temporal dynamics of neural processing in cognition and the development of cell assembly-based models.

TRACE and MultiTrace were developed as modern versions of Hebb's cell assembly construct. The individual parts of each model were theoretically motivated as part of a general cognitive theory. The fact that MultiTrace was able to model the Kleinsmith and Kaplan results with only minor parameter changes lends credence to Kleinsmith and Kaplan's original supposition about the underlying cause of their results. In turn, their data, which has defied other explanations for four decades, shows that the physiology of the brain, including architectural factors such as wiring, are critical in fully understanding human learning.

Hebbian learning has become increasingly popular in connectionist models in recent years and the paradigm explored in this paper provides evidence of how it 
might be useful in a general theory of learning. The postulate that learning comes as the result of correlated neural activity implies that variation in learning can largely be explained in terms of factors that impact that activity. Arousal is a primary example of such a factor. Cell assembly models such as MultiTrace have the potential to explore this idea by modeling the dynamics of neural activity at a fairly high level while still incorporating physiological constraints. These constraints are often architectural, depending on factors that are not easily modeled in many other types of neural networks where, for example, the temporal dynamics of the individual units are essentially irrelevant.

Dynamic models are gaining in popularity, and control issues, such as those involved in the TRACE and MultiTrace models, will be central in their continuing development. Time dependent data, such as the Kleinsmith and Kaplan data is crucial to the development of these models as it provides a source of constraints on how the models must operate. This work is part of an ongoing process of collecting such constraints and using them to develop and calibrate the model. The diversity of the tasks involved - e.g. modeling priming and interference effects in phoneme processing versus modeling paired-associate learning is crucial in showing the generality and power of the model.

\section{Acknowledgments}

This material is based upon work supported by the National Science Foundation under Grant No. 0092605. Any opinions, findings and conclusions or recommendations expressed in this material are those of the author and do not necessarily reflect the views of the National Science Foundation (NSF).

\section{References}

Amit, D.J. (1995). The Hebbian paradigm reintegrated: Local reverberations as internal representations. Behavioral and Brain Sciences, 18(4): 617-657.

Artola, A., \& Singer, W. (1993). Long-term depression of excitatory synaptic transmission and its relationship to long-term potentiation. Trends in Neurosciences, Vol. 16, No. 11, pp. 480-487.

Chown, E. (1994). Consolidation and learning: A connectionist model of human credit assignment. Doctoral dissertation. The University of Michigan.

Eysenck, M.J. (1977). Human Memory: Theory, Research and Individual Differences. Pergamon Press.

Forbell, E. \& Chown, E. (2000). Lexical contact during speech perception: A connectionist model. In Proceedings of the Twenty Second Annual Meeting of the Cognitive Science Society.
Hebb, D.O. (1949). The Organization of Behavior. John Wiley.

Hetherington, P.A., \& Shapiro, M.L. (1993). Simulating Hebb cell assemblies: the necessity for partitioned dendritic trees and a post-not-pre LTD rule. Network, 4, 135-153.

Horn, D., Levy, N., Meilijson, I., \& Ruppin, E. (2000). Distributed synchrony of spiking neurons in a Hebbian cell assembly. In S.A. Solla, T.K. Leen, and K.R. Muller (eds.), Advances in Neural Information Processing Systems, 12, 129-135. MIT Press.

Ito, M. (1992). Posttetanic depression. In L.R. Squire (ed.) Encyclopedia of Learning and Memory. New York: Macmillan Pub. Co.

Kaplan, S. \& Kaplan, R. (1970). The interaction of arousal and retention interval: Ipsative vs normative scoring. Psychonomic Science, 19, 115-117.

Kaplan, S., Sonntag, M. \& Chown, E. (1991). Tracing recurrent activity in cognitive elements (TRACE): A model of temporal dynamics in a cell assembly. Connection Science, 3, 179-206.

Kinsbourne, M. (1982). Hemispheric specialization and the growth of human understanding. American Psychologist, 37(4), 411-420.

Kleinsmith, L.J., \& Kaplan, S. (1963). Paired-associate learning as a function of arousal and interpolated interval. Journal of Experimental Psychology, 65, 190-193.

Kleinsmith, L.J. \& Kaplan, S. (1964). Interaction of arousal and recall interval in nonsense syllable paired-associate learning. Journal of Experimental Psychology, 67, 124-126.

Miller, R.R., \& Marlin, N.A. (1984). The physiology and semantics of consolidation. In H. Weingartner, \& E.S. Parker (Eds.), Memory Consolidation: Psychobiology of Cognition, Hillsdale, NJ: Lawrence Erlbaum.

Oades, R.D. (1985). The role of noradrenaline in tuning and dopamine in switching between signals in the CNS, Neuroscience \& Behavioral Reviews, Vol. 9

Revelle, W. \& Loftus, D.A. (1990). Individual differences and arousal: Implications for the study of mood and memory. Cognition and Emotion, 4, 209237.

Rochester, N., Holland, J.H., Haibt, L.H., \& Duda, W.L. (1956). Tests on a cell assembly theory of the action of the brain, using a large digital computer. IRE Transactions on Information Theory, IT2:80-93.

Sonntag, M.. (1991). Learning sequences in an associative network: A step towards cognitive structure. Doctoral Dissertation. The University of Michigan.

Weingartner, H., \& Parker, E.S. (Eds.) (1984). Memory Consolidation: Psychobiology of Cognition. Hillsdale, NJ: Lawrence Erlbaum. 